\title{
The WinCube Satellite Project Manitoba High School Students Constructing and Launching Pico-Satellite and High Altitude Balloon Payloads
}

\author{
Jeff Cieszecki (VE4CZK) ${ }^{1}$, and Stefan Wagener (VE4NSA) ${ }^{2}$ \\ ${ }^{1}$ Manitoba Satellite Interest Group (MSIG), Co Chair WinCube Satellite Steering Committee; \\ jeff.cieszecki@7oaks.org \\ ${ }^{2}$ Manitoba Satellite Interest Group (MSIG), Co Chair WinCube Satellite Steering Committee; \\ VE4NSA@AMSAT.ORG
}

\begin{abstract}
The WinCube Satellite Project is a cooperative effort among Manitoba high schools, the Manitoba Satellite Interest group (MSIG), the Faculty of Engineering at the University of Manitoba, Maples Collegiate Space Exploration Academy, the Manitoba Aerospace Human Resources Coordinating Committee and numerous aerospace industry partners. Through a mentorship program, Manitoba high school students will design, construct, and launch a pico-satellite with technical support provided by aerospace faculty and engineering students. Basic system design and construction experience for the high school students is provided by the construction and launch of high altitude balloon payloads. Students learn first hand about space mission design, telecommunications, programming, electrical and mechanical engineering.
\end{abstract}

\section{WinCube Satellite Project Concept}

The WinCube Satellite Project was initiated in the Spring of 2006 by the Manitoba Satellite Interest Group (MSIG) and MindSet the Manitoba Network for Science and Technology to provide Manitoba High School Students with the opportunity to be involved in the design, construction, and launching of a picosatellite. Initial funding for the project was obtained through NSERC (Natural Sciences and Engineering Research Council of Canada) and MindSet, the Manitoba Network for Science and Technology as a program of Manitoba Science, Technology, Energy and Mines. The project is designed to challenge students in the fields of science and technology. The pico-satellite is based on the California Polytechnic State University (CalPoly) CubeSat Program design and specifications of a cube satellite with the dimensions of 10x10x10 $\mathrm{cm}$ and a maximum mass of $1 \mathrm{~kg}$. These small, relatively inexpensive satellites are capable of real data gathering as demonstrated by their utilization by universities and space researchers as an economical method of research.

To achieve this lofty goal of creating and launching a pico-satellite, a number of key factors need to be put into place for the high school students:

- Mentorship through aerospace industry and university students

- Gaining experience in payload design and construction through high altitude balloon work (BCube Project)

- Defined educational goals

- Amateur radio certification and ground station operation

For high school students to create a cubesat, the Faculty of Engineering at the University of Manitoba with assistance by Bristol Aerospace provided initial design support for the WinCube satellite. This is necessary to meet the CalPoly specifications for a space worthy satellite. A Preliminary Design Review (PDR) Report was created by a cohort of $4^{\text {th }}$ year Engineering Students at the University of Manitoba during the 2006-2007 school year [1]. The PDR proposes the design of the WinCube satellite and includes: 
- $\quad$ Structure

- $\quad$ Electrical Power

- Communication System

- Command and Data Handling

- Attitude Control

- On Board Science

- $\quad$ Integration and Testing [1]

The "on-board science" [1] will be responsible for gathering scientific data that will be transmitted back to Earth for analysis. The science component of the satellite is what the high school students will contribute directly to. When the WinCube satellite is in orbit, participating high school students will communicate with the satellite via amateur radio. Data gathered will be processed and published by the high school students.

High school students will also take an active role in the construction of the WinCube satellite. Starting in the fall of 2007, students will be mentored by University of Manitoba Engineering Graduate Students during the next phase of construction and testing of the satellite utilizing the new University of Manitoba space lab. To prepare the high school students for the satellite construction, they will gain experience in satellite design by constructing and testing a scientific payload for a high altitude balloon launches. The high altitude balloon work is referred to as BCube.

It is necessary to increase students' basic knowledge of space studies and related concepts before they begin their work on the satellite. Students in the program range in grades from 10 through 12 . It is necessary that students have the basic concepts and vocabulary necessary to understand space science. A "space camp” experience is provided to bridge students' knowledge of high school physics and the concepts and vocabulary they will encounter during their WinCube experience. The "Manitoba Space Adventure" as it is called has been held for two years at the Canadian Forces School of Aerospace Studies, 17 Wing, Canadian Forces Base Winnipeg.

\section{BCube Payload Experience}

The WinCube student design team from the University of Manitoba identified a number of key engineering areas for the WinCube satellite. As stated earlier, the technical expertise required for a space-ready satellite is beyond the skill set for many high school students and since the first year of satellite design was primarily theoretical a real life hands-on project was developed to provide a basic understanding of the intricacies of payload design and fabrication. High School students are given the challenge to create a payload for a high altitude balloon. Some of the similarities between the BCube payload and the WinCube satellite are taken from the Preliminary Design Review (PDR) Report by the University of Manitoba's WinCube Team:

- $\quad$ Payload Frame

o Structure

o Thermal Design

o Passive/Active Thermal Control Systems

- $\quad$ Electrical Power

o Power Budget

o Active/Standby Mode

- Communication

o Requirements

o Amateur Radio Use

o Antennas

- $\quad$ Command and Data Handling [1]

The WinCube satellite once constructed will undergo a number of tests to evaluate its space readiness including exposing the craft to a hard vacuum, extreme temperatures, and vibrations. For the BCube, testing will include physical impacts, cold temperatures, and systems tests. The BCube tests can all be carried out at each high school by the students.

\section{Payload Frame}

The engineering points listed are those that only apply to the BCube design concept. For example, in designing the BCube payload frame, students must take into consideration temperature drops and how the electronics within the payload react as the balloon reaches a potential altitude of $30 \mathrm{~km}$ and external temperature drops as low as $-60^{\circ} \mathrm{C}$.

Areas for consideration in the BCube structure by high school students include the material for the walls (aluminium, foam core, foam insulation) [2], type of adhesives for the structure walls (silicon adhesive, hot glue, Velcro, aluminium tape) [2], and the dimensions of the structure. Students may need to refine payload dimensions to effectively contain all equipment necessary for the balloon launch, yet limit the mass of the structure to the overall $1 \mathrm{~kg}$ payload mass. The design of the BCube structure must also allow for the venting of the internal volume to adjust to external 
atmospheric pressure changes, internal heat loss/gain, and possible moisture damage from clouds [2].

\section{Electrical Power}

High school students will also learn about electrical power budgets in the design of their BCubes. The BCube payloads are expected to operate for 2.5 hours of flight and run an audio beacon upon landing for an extended period of time to aid with ground retrieval. The choice of batteries will also require investigation and testing for suitability.

\section{Communication}

Communication plays a key role in the tracking and data gathering of both the WinCube satellite and the BCube payload. The high school students are required to obtain their amateur radio certificates since balloon to ground communication will utilize amateur radio frequencies similar to those planned for the WinCube satellite. The certification will allow the students to communicate directly with the future WinCube satellite while in orbit via a ground station located in Winnipeg. First hand application of amateur radio operation is done by having the BCube payloads tracked by a GPS radio beacon that is transmitting on 144.390 MHz the official APRS (Automatic Position Reporting System) frequency. The students will use amateur radio transceivers and computer software to track and retrieve the balloon payloads.

\section{Command and Data Handling}

Command and Data Handling of the BCube payloads will be done with Basic Stamp chips. The Basic Stamp is reliable and uses PBasic programming. Students will create programs that meet the needs of their mission designs.

\section{Drop Test}

BCube payloads are tested for impact survival. Students construct a number of prototypes of payloads that contain a mass that places the test container to a total of $1 \mathrm{~kg}$. Payloads are first tested by dropping them off the school roof to evaluate the structures impact survivability. Payloads are then dropped off the roof with various dimensions of parachutes, with time of descents compared. The third drop test involves a drop from an elevated altitude with the use of a kite. The payload is released via a cut down mechanism from the kite, and the payload floats to the ground with the use of a parachute, again recording the time of decent and the altitude it was dropped from. In all of these tests, students are determining the relative strength of the payload structures and best dimension of the parachute design for optimal results versus mass constraints.

\section{Temperature Test}

A key component for any payload is to be able to operate in extreme temperatures. Both the WinCube and BCubes must have measures taken to allow the operation of the electrical system at all times during flight. In the case of the BCube, extreme drops in temperature are an issue, as low as $-60^{\circ} \mathrm{C}$. The BCube payload can utilize either an active or passive thermal control system, to be determined by the students. One possible test for temperature is to place an operational payload in a cooler filled with dry ice [2] or a freezer. In either case, the payloads should be running during the tests [2], with internal and external temperatures of the payload continuously monitored. The tests should run long enough that the BCube reaches thermal equilibrium [2].

\section{Educational Goals}

Educational goals for the WinCube project were developed from the Common Framework of Science Learning Outcomes (Pan-Canadian Framework) written by the Council of Ministers of Education, Canada (CMEC) [3]. General Learning Outcomes (GLOs) from the Pan-Canadian that apply directly to the WinCube Project include Skills, Communication and Teamwork.

Pan-Canadian GLO for Skills includes planning investigations to record and analyse data using a variety of techniques [3]. For the BCube payload design or the science component of the WinCube satellite, students will develop the function of these payloads while attempting to address a scientific issue.

The GLO for Communication and Teamwork involves the effective communication with others in regards to issues and ideas, and come up with a strategy that has a consensus to move forward on [3]. For the BCube component, students will work as a team to design a payload within a high school. The WinCube satellite will require students from participating high schools to discuss and agree upon what the science component will accomplish once in orbit. 
Specific Learning Outcomes (SLOs) from the PanCanadian Framework found in the WinCube Project include Initiating and Planning; Performing and Recording; Analyzing and Interpreting [3].

Initial BCube payload designs will be very basic, to simply launch and retrieve a payload. Subsequent payload designs will have more specific scientific goals. The SLO of Initiating and Planning involves the investigation of practical problems and issues and the creation of scientific investigations to gather data [3]. The SLO of Performing and Recording will have students carry out experiments while controlling variables, effectively collecting and compiling data [3]. With data collected, students will analyze evidence, provide conclusions, and display information using a variety of formats as part of the SLO Analyzing and Interpreting [3].

\section{Amateur Radio and High Powered Rocketry}

Further educational opportunities include certification in amateur radio and potentially high powered rocketry. During the 2006-2007 school year, participating high school students took part in classes for amateur radio certification. Amateur radio is a key component of the WinCube project that allows students to track their BCube payloads and communicate with the WinCube satellite while in orbit. The amateur radio course will again be offered during the 2007-2008 school year.

A planned additional course to be offered to participating students in the fall of 2007 is a junior certification in high powered rocketry. The rocketry course will provide students with a better understanding of the physics of launching payloads into space.

\section{Conclusion}

The WinCube project is a unique attempt allowing high school students to be directly involved in the process of designing, building, testing and operating a fully functional satellite (WinCube). The success of this multi year project depends on strong partnerships with the educational, private and governmental sector. A high altitude balloon project was initiated to provide the high school students with hands-on experience in the design and operation of payloads.

\section{References}

[1] Shambrock, J., W. Whaley, B. Klimenko, P. Wheatley, D. Boyd, S. Tully, J. Eady, C. Bosecke, J. LaRue, T. vanBeek, R. Le Neal., University of Manitoba Win-Cube Project 2006-2007 Preliminary Design Review, Winnipeg, Manitoba, 2007.

[2] Koehler, Chris, "BalloonSat: Mission to the Edge of Space”, Proceedings of the $16^{\text {th }}$ Annual/USU Conference on Small Satellites, Logan, Utah, August, 2002.

[3] Council of Ministers of Education, Canada (CMEC), Common Framework of Science Learning Outcomes: PanCanadian Protocol for Collaboration on School Curriculum, Council of Ministers of Education, Canada, Toronto, 1997. 
